\title{
Validation of the qi blood yin yang deficiency questionnaire on chronic fatigue
}

\author{
Jihye Kim, Boncho Ku and Keun Ho Kim*
}

\begin{abstract}
Background: Chronic fatigue (CF) reflects an imbalance of inter-organ functions or of the four essential physiological components qi, blood (xue), yin, and yang. CF can be subdivided into different patterns. However, there are no diagnostic methods for CF. This study aimed to clinically validate a pattern identification method by identifying correlations between CF and responses to the gi blood yin yang deficiency questionnaire (QBYY-Q).

Methods: Participants were recruited between May and June 2014 through the Kyung Hee University Korean Medicine hospital website and via posters and comprised 129 CF patients diagnosed with the United States Centers for Disease Control and Prevention (1994) criteria. Participants who had organic diseases that explained the CF were excluded. A total of 159 participants were asked to complete the QBYY-Q, the fatigue severity scale, and the Chalder fatigue scale. The latter two questionnaires were used to assess convergent validity with the QBYY-Q. Among the 129 CF participants, 70 and 59 had chronic fatigue syndrome and idiopathic chronic fatigue, respectively. Two Korean medical doctors independently assessed participants' qi, blood, yin, and yang deficiency patterns using QBYY deficiency pattern identification guidelines. Based on the results of a preliminary study of the QBYY-Q, we selected 32 reliable items for symptoms corresponding to each deficiency pattern. The items were used to estimate internal consistency and construct validity. Multinomial logistic regression analysis was performed for scores on each deficiency pattern.

Results: The data were means and standard deviations or numbers of participants and proportions for continuous and categorical variables, respectively. A statistical significance level of $P<0.05$ was assumed. The QBYY-Q showed satisfactory internal consistency. Explanatory factor analysis extracted two factors for each deficiency pattern. The percentages of explained variance for qi, blood, yin, and yang deficiency were 45.1, 58.0, 52.2, and $63.4 \%$, respectively. Each QBYY-Q deficiency score was positively associated with each corresponding deficiency pattern. Qi deficiency was used as a reference category. Odds ratios of blood, yin, and yang deficiency were 10.97, 10.69, and 14.64, respectively.
\end{abstract}

Conclusion: The QBYY-Q was suitable for estimating the influences of qi, blood, yin, and yang deficiencies in CF. Trial registration This trial was registered with the Korean Clinical Trial Register (KCT0001199)

Keywords: Pattern identification, Deficiency pattern, Questionnaire, Qi blood yin yang deficiencies, Chronic fatigue

\section{Background}

Fatigue is a state of subjective tiredness and can be subdivided into prolonged fatigue and chronic fatigue (CF). $\mathrm{CF}$ is a condition of subjective tiredness and is reported by nearly $10 \%$ of the global population [1]; it can be categorized as either explained CF or unexplained CF.

*Correspondence: rkim70@kiom.re.kr

KM Fundamental Research Division, Korea Institute of Oriental Medicine,

1672 Yuseongdae-ro, Yuseong-gu, Daejeon 34054, Republic of Korea
Unexplained CF is further subdivided into chronic fatigue syndrome (CFS) and idiopathic chronic fatigue (ICF) [2]. CFS is characterized by severe disabling fatigue and a combination of four additional symptoms that may include impairments in cognitive or neurological function, sleep dysfunction, musculoskeletal pain, and endocrine or immune dysfunction (Table 1). Additionally, alternative medical and psychiatric causes must have been excluded, and the condition must have persisted 
Table 1 Diagnosis CDC-1994 criteria

\begin{tabular}{l} 
Chronic fatigue syndrome symptoms \\
\hline A. Major criteria (both criteria required) \\
1. Severe fatigue $>6$ months \\
a. Persistent or relapsing in course \\
b. Does not resolve with bed rest \\
c. Significant reduction in average daily activity (below $50 \%$ of prior \\
activity) \\
2. Other causes excluded \\
a. See fatigue causes \\
B. Minor criteria (4 or more present for 6 months) \\
1. Headache \\
a. New type, severity or pattern \\
b. Typically non-focal headache \\
2. Migratory polyarthralgias \\
a. Non-inflammatory (no swelling or erythema) \\
3. Myalgias \\
4. Postexertional malaise or fatigue \\
a. Duration > $24 \mathrm{~h}$ \\
5. Short-term memory or concentration impaired \\
6. Pharyngitis \\
7. Tender cervical or axillary adenopathy \\
8. Non-restorative sleep
\end{tabular}

for at least 6 months. ICF meets all the criteria for CFS except for the four additional symptoms.

Studies of CFS and ICF indicate prevalence rates of approximately 10 and $1 \%$ of the general population, respectively [3]. The estimated prevalence of CF in the Republic of Korea is between 0.6 and $2 \%$ of the general adult population [4]. Several hypotheses have been proposed for the pathogenesis of $C F$, including oxidative stress, hypothalamic-pituitary-adrenal axis abnormalities, and immune dysfunction [5-7]. Western medicine offers no objective diagnostic methods or effective therapies for $\mathrm{CF}$ and the recommended drugs have many side effects [8-11]. CFS and ICF patients tend to be interested in complementary and alternative medicine treatments $[9,12,13]$.

An individual's constitution and current health status can be conceptualized in terms of the balance between the $q i$ and blood (xue) conditions of the internal organ functions and of the co-existing yin and yang (which constitute the physical form and functioning of the human body and its deficient and excessive energetic qualities) $[9,14,15]$. Treatments such as acupuncture, moxibustion, herbal medicine, and qigong restore the balance of the four essential components of the human body: qi, blood, yin, and yang.

Unexplained CF can be classified as either a deficiency syndrome or an excess syndrome; these syndromes refer to the deficiency or excess of qi, blood, yin, and yang [16, 17]. Unexplained CF is usually considered a deficiency pattern representing an imbalance of the four essential components; most cases are treated by invigorating $q i$ and yang and nourishing blood and yin $[9,18,19]$. CF treatments are based on individual patterns of body constitution or pattern identification (PI); most patients are diagnosed according to four patterns of body constitution that involve the five major organs: the heart, liver, spleen, lungs, and kidney. These patterns are qi deficiency (QD, i.e., decreases in visceral functions and body resistance), blood deficiency (BD, i.e., failure to nourish the organs, tissues, and meridians), yin deficiency and yang deficiency ( $\mathrm{YnD}$ and $\mathrm{YgD}$, i.e., failure to maintain normal function of internal organs) $[9,11,18]$.

This study aimed to clinically validate a PI diagnostic method by testing for correlations between CF and responses to the qi blood yin yang deficiency questionnaire (QBYY-Q).

\section{Methods \\ Ethics}

The study protocol was reviewed and approved by the Institutional Review Board of the Cheonan Oriental Hospital at Daejeon University (authorization number: 2014A01-02) and was registered with the clinical research information service (registration number: KCT0001199) (Additional file 1).

\section{Study design}

This study was designed as a single-center, cross-sectional, case-controlled study, and it was performed at the Cheonan Oriental Hospital of Daejeon University in Cheonan, South Korea from May to June 2014. The procedures of this study were depicted in Fig. 1.

\section{Participants}

Participants were recruited by advertising on hospital websites and posters displayed in hospitals and local universities, Seoul, Republic of Korea from May to June 2014. They went through telephone pre-screening by the clinical study coordinator to ensure that CF patients met the inclusion criteria of "males and females aged 19-39" and "the presence of $\mathrm{CF}$ that is continuous and repetitive for more than 6 months". Then, participants visited the clinical study center and go through a more in-depth screening process to determine whether they satisfied the inclusion criteria.

Participants who worked at night or drank alcohol more than twice per week were excluded. We excluded the participants who had organic diseases for explained CF, such as acute or chronic liver disease (e.g., hepatitis, and liver cirrhosis); anemia; tuberculosis; chronic lung 


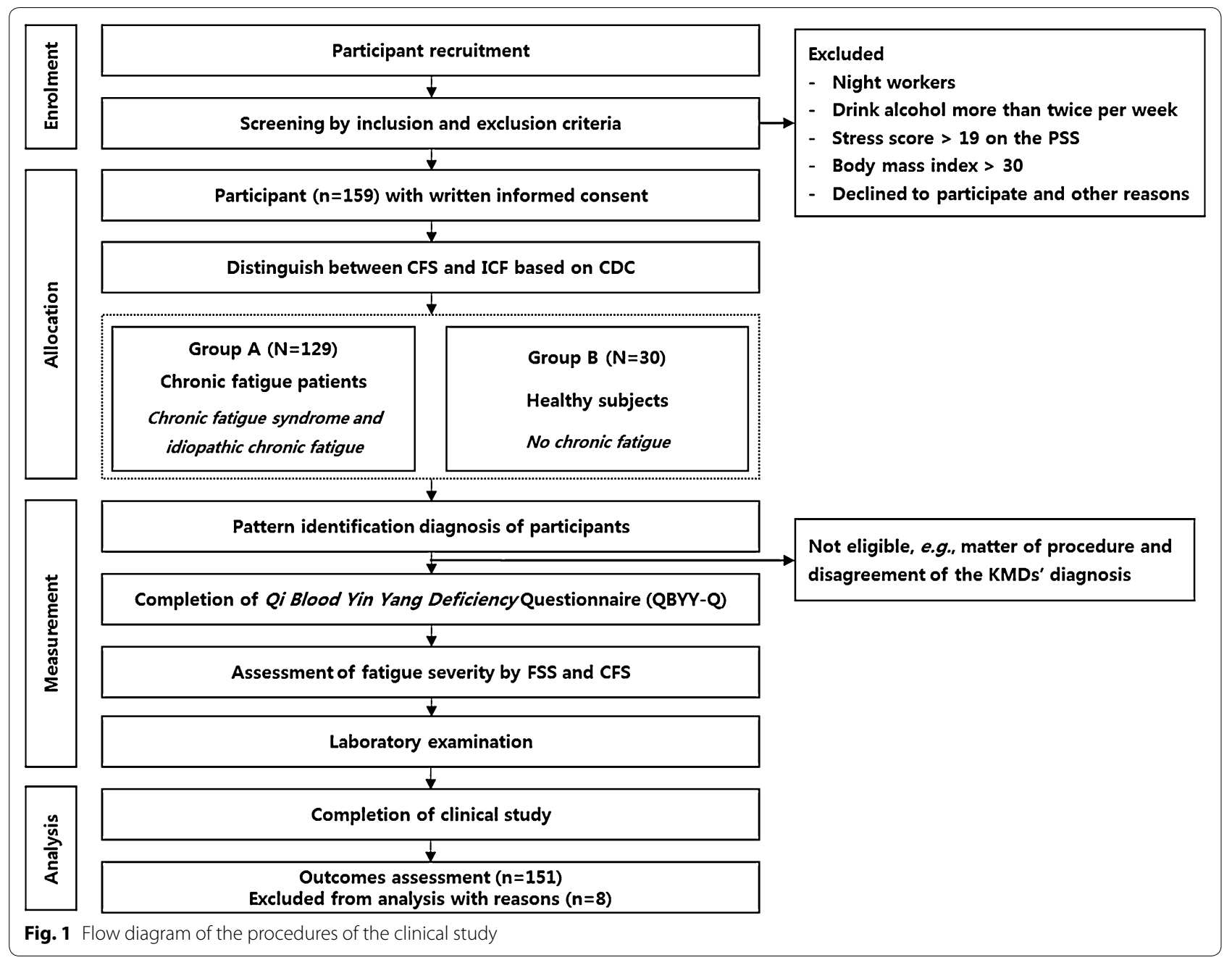

disease; cardiovascular disease (e.g., heart failure, hypertension); endocrine/metabolic disease (e.g., diabetes, thyroid gland disease, and severe obesity); autoimmune disease (e.g., rheumatoid arthritis, systemic lupus erythematosus, and multiple sclerosis); malignant tumors; or infectious disease were excluded. In addition, CF patients with psycho-social diseases, such as depression, anxiety neurosis, recent severe stress, schizophrenia, alcoholism, or an eating disorder (e.g., anorexia nervosa, bulimia nervosa), were excluded $[12,13]$. Cut-offs of BMI $(\geq 30 \mathrm{~kg} /$ $\mathrm{m}^{2}$ ) have shown good specificity in diagnosing severe obesity, which is defined as BMI $\geq 30 \mathrm{~kg} / \mathrm{m}^{2}$ based on measured weight and height and is a type of metabolic disease $[20,21]$. The participants were informed of the entire process of the clinical study and were asked to sign informed consent forms (Additional file 2).

The final participants included 129 patients with unexplained CF and 30 controls. The enrolled patients mainly suffered from fatigue lasting more than 6 months. The CDC-1994 criteria for CFS [22] were used to distinguish between CFS and ICF, using the cutoff of 4 among the 8 symptoms in the minor criteria, respectively (Table 1 ). CFS patients complained of major criteria symptoms and more than four of the minor criteria symptoms, and ICF patients complained of major criteria symptoms and less than four of the minor criteria symptoms. If a subject complained of fatigue lasting more than 6 months, headache and pharyngitis, the subject was diagnosed as ICF. Among the attending $129 \mathrm{CF}$ patients, 70 and 59 were found to have CFS and ICF, respectively.

The control (30 healthy participants) came to the same clinic who worked at night and drank alcohol were excluded. The control group consisted of participants who satisfied the following criteria: (1) not meeting the CFS and ICF criteria from CDC-1994; (2) absence of organopathy; and (3) normal results of laboratory tests and radiological examinations.

Of the 159 participants, two participants were excluded due to noncompliance with the process described in the protocol; therefore, 157 participants were included in this study. 


\section{Pattern diagnosis}

There is no "gold standard" for pattern diagnosis. Our previous study demonstrated discrepancies in the diagnoses of QD, BD, YnD, and YgD among Korean medical doctors (KMDs) [23]. To increase the internal consistency of KMD expert pattern diagnosis, we created PI guidelines for $\mathrm{QD}, \mathrm{BD}, \mathrm{YnD}$, and $\mathrm{YgD}$ with PI experts, using data from previous studies $[9,24]$ and the standard Korean medical literature [22] 4 months before the study commenced. The developed PI guidelines were validated after consultation with experts. Based on the PI guidelines, two KMDs with 4 years of clinical experience independently diagnosed the participants' deficiency status; the KMDs were blinded to the results of the diagnoses. Participants for whom the KMD diagnoses disagreed were excluded from the analysis.

\section{Measurements \\ QBYY-Q scales}

In our previous studies, items related to QD, YnD, YgD, and $\mathrm{BD}$ were systematically reviewed [9, 25-27], and a QBYY-Q constructed by a panel of experts was selected for use in a pilot study. This QBYY-Q had been established by an expert panel of $27 \mathrm{KMDs}$ in a previous study [28]. A pilot study was performed to determine the reliability and validity of the QBYY-Q for 100 participants with CF [23]. After these participants completed the QBYY-Q, the KMDs divided the patients into four groups: QD, BD, YnD, and YgD. After 3 weeks, the 100 participants were retested in the same way. The results from our pilot study showed that Cronbach's $\alpha$ coefficient was 0.916 for internal consistency of the QBYY-Q. Construct validity analyzed using exploratory factor analysis (EFA) produced four factors with eigenvalues greater than 1.0. Factors 1, 2, 3, and 4 were identified as QD, $\mathrm{YnD}$, $\mathrm{YgD}$, and $\mathrm{BD}$, respectively. However, four items, "menstrual cycle slowdown," "decreased masculine energy," "damp and cold penis," and "decreased sexual desire," produced an item-total correlation of less than 0.5. Because the results indicated that the four items were not equivalent to the others, these items were excluded from the validity tests. Test-retest reliability was high (intraclass correlation coefficient $=0.699$ ) [23]. Consequently, the final version of the QBYY-Q included 32 sign and symptom entries consisting of nine $\mathrm{QD}$, eight $\mathrm{BD}$, nine $\mathrm{YnD}$, and six YgD pattern items. The order of the $32 \mathrm{QBYY}-\mathrm{Q}$ pattern items was reversed to reduce bias (Additional file 3). The QD pattern included two signs, enervation and shortage of $q i$ or faint breathing, and the BD pattern included three signs, pale complexion, sweating during the day, and palpitations (a subjective sensation of rapid and forceful beating of the heart). The YnD pattern was divided into following two signs: (1) dry skin and mouth; and (2) heat vexation and fever accompanied by uneasiness or restlessness. The YgD pattern was divided into two signs, cold in the extremities up to the knees and elbows and diarrhea. The severity of each item was graded using the following 4-point scale: $1=$ strongly disagree, $2=$ disagree, $3=$ agree, and $4=$ strongly agree. The scores for each item were totaled, and QD-Q, BD-Q, YnD-Q, and YgD-Q scores calculated.

\section{Fatigue severity scale (FSS)}

The FSS is a self-rating questionnaire comprising nine items that are scored between 1 (completely disagree) and 7 (completely agree). The items assess the extent of fatigue symptoms and their impact on patient functioning (including motivation; exercise; physical functioning; ability to perform duties; and interference with work, family, or social life) [29]. Example items on the questionnaire are "exercise brings on my fatigue" and "my fatigue is very debilitating"; higher item scores indicate a greater degree of fatigue.

\section{Chalder fatigue scale-Korean version (CFS-K)}

The CFS is a self-reported questionnaire that measures fatigue intensity on a 4-point scale $(0=$ less than usual to $3=$ much more than usual) [30]. Symptoms assessed by the scale are divided into two subcategories: seven items related to physical symptoms, and four items related to mental fatigue. Although the internal consistency of the CFS-K has not been fully reported, many studies using versions of the CFS in different languages have shown strong internal consistency for both the physical and mental symptoms of fatigue. Likert scoring with weights was used to score the magnitude of fatigue [30].

\section{Statistical analysis}

The data were represented as means \pm standard deviations (SDs) or as numbers of participants and proportions for continuous and categorical variables, respectively. All statistical analyses were performed using $\mathrm{R}$ software (The $\mathrm{R}$ Foundation), version 3.1.1, on a Windows 7 platform. $\mathrm{R}$ software is a free software environment for statistical computing and graphics [31]. The level of significance was set at $P<0.05$ for all analyses. Inter-rater agreement for the two KMDs' diagnoses of QD, BD, YnD, and YgD was evaluated using Cohen's $\mathrm{k}$ coefficient. Differences between CF patients (CFS and ICF) and the non-CF group were tested using a one-way ANOVA for continuous variables and Pearson's Chi square test for categorical variables. The Keiser-Meyer-Olkin (KMO) test was used to test sample adequacy (considered acceptable if the KMO constant is >0.60) [32]. The KMO was used to check the matrix of correlations for the applicability of factorial analysis, and then a principal axis factoring 
extraction method was applied. Construct validity for each deficiency scale on the questionnaire was estimated by performing EFA with a combination of minimum residual extraction and promax rotation. For each deficiency scale, conceptual factors were identified by examining the factor loadings. The internal consistency of each deficiency scale was also tested using Cronbach's $\alpha$ coefficient. Convergent validity of deficiency scores derived from the four QBYY-Q scales and the total scores on the CFS-K and FSS was assessed using Spearman's correlation coefficient. The mean differences in deficiency scores between the CF and non-CF groups were examined using a one-way ANOVA test. In addition, binary logistic regression analysis was used to estimate the odds ratios (ORs) of CF (CFS + ICF versus no CF) in the KMD diagnoses of $\mathrm{QD}, \mathrm{BD}, \mathrm{YnD}$, and $\mathrm{YgD}$ to confirm the associations between CF and PI. Both CF and PI could have been influenced by other confounding factors; therefore, age and sex were included to estimate the adjusted ORs for CF. Discriminative validity was determined using the ORs of the four deficiency scale scores derived from a multinomial logistic regression model.

\section{Results}

\section{Demographic characteristics}

Because of the absence of a gold standard for pattern diagnosis, six of the 157 participants showed discrepancies in pattern diagnoses and were excluded to increase the internal consistency. Therefore, 151 participants were included in the analysis. Table 2 shows the demographic characteristics of the 65 participants with CFS, the 56 participants with ICF, and the 30 control subjects in the non-CF group. For most of the characteristics, there were no significant differences among the CF groups (CFS and ICF) and the control group. However, the age and sex distributions showed significant differences among the three groups. The means and SDs for age were $29.7 \pm 4.9$ years for participants with CFS, $27.4 \pm 5.2$ years for participants with ICF, and $26.0 \pm 3.7$ years for the control group $(F=7.09, P=0.001)$. The proportions of women in the CFS, ICF, and control groups were 64.6, 35.7, and $50.0 \%$, respectively $\left(X^{2}=10.07, P=0.007\right)$.

\section{KMD inter-rater agreement}

Cohen's $\mathrm{k}$ coefficient was $0.95(\mathrm{Z}=20.69, P<0.001)$, which indicated that the agreement between the two KMDs was sufficiently strong. The two KMDs differed in their PI diagnoses for six (3.8 \%) of the 157 participants (Table 3).

Internal consistency and construct validity of the QBYY-Q The internal consistency and construct validity of each of the QBYY-Q scales were assessed by merging the current
Table 2 Demographic characteristics of participants with CF (CFS and ICF) and controls

\begin{tabular}{|c|c|c|c|c|}
\hline & \multicolumn{2}{|c|}{ Chronic fatigue } & \multirow{2}{*}{$\begin{array}{l}\text { Controls } \\
(n=30)\end{array}$} & \multirow[t]{2}{*}{$P$ value } \\
\hline & CFS $(n=65)$ & $\operatorname{ICF}(n=56)$ & & \\
\hline Age (years) & $29.7 \pm 4.9$ & $27.4 \pm 5.2$ & $26.0 \pm 3.7$ & 0.001 \\
\hline Sex (female) & $42(64.6)$ & $20(35.7)$ & $15(50.0)$ & 0.007 \\
\hline BMI $\left(\mathrm{kg} / \mathrm{m}^{2}\right)$ & $22.8 \pm 3.5$ & $22.3 \pm 3.0$ & $21.8 \pm 3.1$ & 0.746 \\
\hline $\mathrm{SBP}(\mathrm{mm} \mathrm{Hg})$ & $116.3 \pm 11.0$ & $116.8 \pm 10.2$ & $119.2 \pm 12.7$ & 0.471 \\
\hline $\mathrm{DBP}(\mathrm{mm} \mathrm{Hg})$ & $69.5 \pm 11.1$ & $71.8 \pm 9.0$ & $71.2 \pm 12.5$ & 0.471 \\
\hline $\begin{array}{l}\text { Pulse rate } \\
\text { (beats/min) }\end{array}$ & $71.4 \pm 8.9$ & $70.0 \pm 6.3$ & $72.0 \pm 9.5$ & 0.482 \\
\hline $\begin{array}{l}\text { Body tempera- } \\
\text { ture }\left({ }^{\circ} \mathrm{C}\right)\end{array}$ & $36.4 \pm 0.2$ & $36.4 \pm 0.2$ & $36.4 \pm 0.2$ & 0.601 \\
\hline Smoking & $18(27.7)$ & $13(23.2)$ & $4(13.3)$ & 0.305 \\
\hline $\begin{array}{l}\text { Regular } \\
\text { exercise }\end{array}$ & $20(30.8)$ & $27(48.2)$ & $12(40)$ & 0.145 \\
\hline Regular diet & $36(55.4)$ & $33(58.9)$ & $19(63.3)$ & 0.76 \\
\hline \multicolumn{5}{|c|}{ Pattern identification } \\
\hline QD & $16(24.6)$ & $13(23.2)$ & $14(46.7)$ & 0.129 \\
\hline $\mathrm{BD}$ & $13(20)$ & $18(32.1)$ & $6(20)$ & \\
\hline$Y n D$ & $16(24.6)$ & $14(25)$ & $6(20)$ & \\
\hline YgD & $20(30.8)$ & $11(19.6)$ & $4(13.3)$ & \\
\hline
\end{tabular}

The data are represented as the means \pm SDs or as numbers of participants and proportions; $\mathrm{N}(\%)$ for continuous and categorical variables, respectively. Oneway ANOVA was performed for continuous variables and Pearson's $X^{2}$ test for categorical variables

CFS chronic fatigue syndrome, ICF idiopathic chronic fatigue, SBP systolic blood pressure, DBP diastolic blood pressure, QD qi deficiency, BD blood deficiency, $Y n D$ yin deficiency, YgD yang deficiency

Table 3 Agreement results in the KMD diagnoses $(n=157)$

\begin{tabular}{|c|c|c|c|c|c|}
\hline \multicolumn{2}{|c|}{ KMD diagnoses } & \multicolumn{4}{|c|}{ KMD \#1 } \\
\hline & & QD & BD & YnD & $\mathrm{YgD}$ \\
\hline \multirow[t]{4}{*}{ KMD \#2 } & QD & 43 & 0 & 0 & 1 \\
\hline & $\mathrm{BD}$ & 1 & 37 & 1 & 0 \\
\hline & $\mathrm{YnD}$ & 1 & 2 & 36 & 0 \\
\hline & $\mathrm{YgD}$ & 0 & 0 & 0 & 35 \\
\hline
\end{tabular}

KMD Korean medical doctor, QD qi deficiency, BD blood deficiency, $Y n D$ yin deficiency, YgD yang deficiency

study and pilot study data (totaling 252 cases). The Cronbach's $\alpha$ coefficients of the nine QD items (QD-Q), the eight $\mathrm{BD}$ items (BD-Q), the nine YnD items (YnD-Q), and the six YgD items (YgD-Q) were 0.816, 0.826, 0.807, and 0.717 , respectively.

KMO tests of sphericity confirmed that each QBYY$\mathrm{Q}$ scale contained an eligible number of items and a sufficient sample size $(\mathrm{QD}-\mathrm{Q}=0.856, \mathrm{BD}-\mathrm{Q}=0.833$, YnD-Q $=0.807$, YgD-Q $=0.702$ ). Two factors were extracted for each QBYY-Q scale following EFA with 
minimum residual extraction and promax rotation. The EFA results for each QBYY-Q scale are shown in Table 4.

\section{Convergent validity of the QBYY-Q}

The convergent validity of each QBYY-Q scale was assessed with the FSS and CFS-K using pairwise Spearman's correlation coefficients (Table 5). Total deficiency scores for the QD-Q, BD-Q, YnD-Q, and YgD-Q were calculated by averaging all the items corresponding to each deficiency scale. Subscores for each deficiency scale were also obtained by averaging the items relevant to each factor, which were identified from the EFA. The QD-Q score correlated significantly with both the FSS $(r=0.721$, $P<0.001)$ and the CFS-K $(r=0.645, P<0.001)$. The BD-Q score also correlated significantly with the FSS

Table 4 Extracted factor loadings of items for each QBYY-Q and the variance explained by two factors

\begin{tabular}{|c|c|c|c|c|c|c|c|c|c|c|}
\hline \multirow[t]{2}{*}{ PI } & \multirow[t]{2}{*}{ Item } & \multirow[t]{2}{*}{ Sub-scale } & \multicolumn{2}{|l|}{ QD-Q } & \multicolumn{2}{|l|}{ BD-Q } & \multicolumn{2}{|l|}{ YnD-Q } & \multicolumn{2}{|l|}{ YgD-Q } \\
\hline & & & FL1 & FL2 & FL1 & FL2 & FL1 & FL2 & FL1 & FL2 \\
\hline \multirow[t]{10}{*}{ QD } & QD-Q 01 & Fatigue & 0.821 & -0.054 & & & & & & \\
\hline & QD-Q 02 & & 0.798 & -0.087 & & & & & & \\
\hline & QD-Q 05 & & 0.824 & -0.033 & & & & & & \\
\hline & QD-Q 06 & & 0.444 & 0.043 & & & & & & \\
\hline & QD-Q 07 & & 0.367 & -0.107 & & & & & & \\
\hline & QD-Q 09 & & 0.287 & 0.160 & & & & & & \\
\hline & QD-Q 03 & Weakened voice & -0.147 & 1.098 & & & & & & \\
\hline & QD-Q 04 & & 0.231 & 0.330 & & & & & & \\
\hline & QD-Q 08 & & 0.171 & 0.433 & & & & & & \\
\hline & $\%$ of varian & explained & 27.90 & 17.20 & & & & & & \\
\hline \multirow[t]{9}{*}{$\mathrm{BD}$} & BD-Q 01 & Heart pounding & & & 0.831 & -0.081 & & & & \\
\hline & BD-Q 02 & & & & 0.865 & -0.182 & & & & \\
\hline & BD-Q 03 & & & & 0.537 & 0.098 & & & & \\
\hline & BD-Q 08 & & & & 0.537 & 0.016 & & & & \\
\hline & BD-Q 04 & Pale and dry body & & & 0.112 & 0.499 & & & & \\
\hline & BD-Q 05 & & & & 0.090 & 0.617 & & & & \\
\hline & BD-Q 06 & & & & -0.222 & 0.977 & & & & \\
\hline & BD-Q 07 & & & & 0.308 & 0.313 & & & & \\
\hline & $\%$ of variar & explained & & & 45.30 & 12.70 & & & & \\
\hline \multirow[t]{10}{*}{$Y n D$} & YnD-Q 02 & Rough and dry body & & & & & 0.508 & 0.145 & & \\
\hline & YnD-Q 05 & & & & & & 0.712 & -0.120 & & \\
\hline & YnD-Q 06 & & & & & & 0.770 & -0.102 & & \\
\hline & YnD-Q 07 & & & & & & 0.689 & -0.033 & & \\
\hline & YnD-Q 01 & Body steaming & & & & & 0.243 & 0.267 & & \\
\hline & YnD-Q 03 & & & & & & -0.042 & 0.741 & & \\
\hline & YnD-Q 04 & & & & & & 0.216 & 0.448 & & \\
\hline & YnD-Q 08 & & & & & & -0.154 & 0.848 & & \\
\hline & YnD-Q 09 & & & & & & 0.054 & 0.388 & & \\
\hline & $\%$ of varian & explained & & & & & 39.60 & 12.60 & & \\
\hline \multirow[t]{7}{*}{ YgD } & YgD-Q 01 & Preference for warm things & & & & & & & 0.821 & -0.120 \\
\hline & YgD-Q 02 & & & & & & & & 0.560 & 0.012 \\
\hline & YgD-Q 03 & & & & & & & & 0.692 & -0.072 \\
\hline & YgD-Q 04 & & & & & & & & 0.417 & 0.225 \\
\hline & YgD-Q 05 & Diarrhea & & & & & & & -0.146 & 0.939 \\
\hline & YgD-Q 06 & & & & & & & & 0.052 & 0.646 \\
\hline & $\%$ of varian & explained & & & & & & & 41.50 & 21.90 \\
\hline
\end{tabular}

The italic letters represents the largest loading between two factors

$P I$ pattern identification, $Q D$ qi deficiency, $Q D-Q$ qi deficiency questionnaire, BD blood deficiency, BD-Q blood deficiency questionnaire, $Y n D$ yin deficiency, YnD- $Q$ yin deficiency questionnaire, $Y g D$ yang deficiency, $Y g D$ - $Q$ yang deficiency questionnaire, $F L$ factor loading 
Table 5 Pairwise Spearman's correlation coefficients of QBYY-Q scores and sub-scales of the QD-Q, BD-Q, YnD-Q and YgD-Q with the CFS-K and FSS

\begin{tabular}{lcccc}
\hline & CFS-K & $P$ value & FSS & $P$ value \\
\hline QD-Q score & 0.645 & $<0.001$ & 0.721 & $<0.001$ \\
QD-Q S1: Fatigue & 0.653 & $<0.001$ & 0.704 & $<0.001$ \\
QD-Q S2: Weakened voice & 0.419 & $<0.001$ & 0.484 & $<0.001$ \\
BD-score & 0.467 & $<0.001$ & 0.441 & $<0.001$ \\
BD-Q S1: Heart pounding & 0.298 & $<0.001$ & 0.366 & $<0.001$ \\
BD-Q S2: Pale and dry body & 0.481 & $<0.001$ & 0.411 & $<0.001$ \\
YnD-score & 0.485 & $<0.001$ & 0.408 & $<0.001$ \\
YnD-Q S1: Rough and dry body & 0.387 & $<0.001$ & 0.252 & 0.0018 \\
YnD-Q S2: Body steaming & 0.411 & $<0.001$ & 0.433 & $<0.001$ \\
YgD-score & 0.291 & $<0.001$ & 0.403 & $<0.001$ \\
YgD-Q S1: Preference for warm & 0.295 & $<0.001$ & 0.384 & $<0.001$ \\
things & & & & \\
YgD-Q S2: Diarrhea & 0.083 & 0.314 & 0.152 & 0.063 \\
\hline
\end{tabular}

CFS-K Korean version of the Chalder fatigue scale, FSS fatigue severity scale, QDscore qi deficiency score, BD-score blood deficiency score, YnD-score yin deficiency score, $Y g D$-score yang deficiency score

$(r=0.44, P<0.001)$ and the CFS-K $(r=0.47, P<0.001)$. The YnD-Q score correlated significantly with the FSS $(r=0.41, P<0.001)$ and the CFS-K $(r=0.48, P<0.001)$. The YgD-Q score also correlated significantly with the FSS $(r=0.40, P<0.001)$ and the CFS-K $(r=0.29$, $P<0.001)$. Most of the subscales for the deficiency scales were significantly correlated with both the CFS-K and the FSS, with correlations ranging from 0.295 to 0.704 . However, YgD-S2 was not significantly correlated with either the CFS-K $(r=0.083, P=0.314)$ or the FSS $(r=0.152$, $P=0.063)$.

\section{Least square means of QBYY-Q scores with CF status}

The least square means of each QBYY-Q scale score with CF status were acquired after multivariate adjustment for sex and age. Each marginal mean corresponding to a QBYY-Q score was estimated from the linear mixed model. The least square means of each QBYY-Q scale for the $\mathrm{CF}$ and the control groups are shown in Fig. 2a. The total scores for the QD-Q, BD-Q, YnD-Q, and YgD-Q for the CFS patients were 1.962, 2.038, 1.928, and 1.767, respectively, and those for the control group were $1.615,1.554,1.530$, and 1.411 , respectively. Patients with a diagnosis of CFS showed significantly higher QD, $\mathrm{BD}$, and $\mathrm{YnD}$, and $\mathrm{YgD}$ scores than those diagnosed with ICF (QD-Q score: $P<0.001$; BD-Q score: $P=0.001$, YnD-Q score: $P=0.002$; YgD-Q score: $P=0.024$ ) or those in the control group (QD-Q score: $P=0.042$; BD-Q score: $P=0.001$; YnD-Q score: $P=0.002$; YgD-Q score: $P=0.008$ ). The profiles of the mean QBYY-Q scores among the control, ICF, and CFS groups are shown in Fig. 2b. The mean difference between the QD score and $\mathrm{YgD}$ score was significant for both the control $(P=0.042)$ and the CFS group $(P=0.001)$. There were no significant differences among QBYY-Q scores for the ICF group.

\section{Association between $\mathrm{PI}$ and $\mathrm{CF}$}

To analyze the association between the KMD diagnosis and CF, the ICF and CFS groups were merged into one
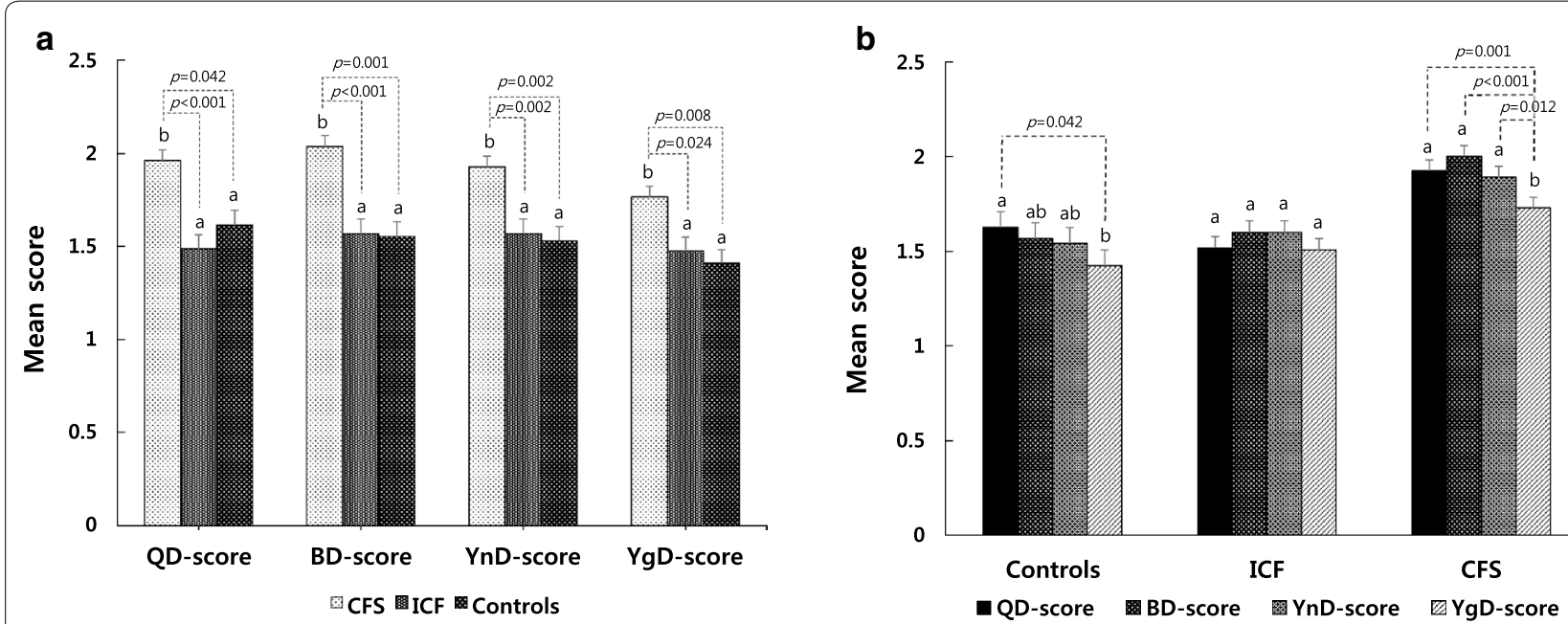

Fig. 2 Least mean square plot for QBYY-Q scores. a Least mean squares of each QBYY-Q score according to the control, ICF, and CFS groups; b Least mean squares of QBYY-Q scores among the control, ICF, and CFS groups after adjustment for sex and age. Dotted lines denote the significant pairwise comparisons in which the $P$ value was less than 0.05 . Alphabetic notations (i.e., $a$, ab and b) above the standard error bars indicate the homogeneous groups resulting from Tukey's HSD test for multiple comparisons 
large CF group. Table 6 shows the crude and adjusted ORs for the association between the KMD PI diagnoses and $\mathrm{CF}$, which were acquired through binomial logistic regression. The crude $\mathrm{ORs}$ for $\mathrm{BD}, \mathrm{YnD}$, and $\mathrm{YgD}$ were significantly higher than the OR for QD (2.49-, 2.41-, and 3.74-fold, respectively). The prevalence ORs of BD, $\mathrm{YnD}$, and $\mathrm{YgD}$ in model 1 were 3.41-, 3.41-, and 4.68-fold higher, respectively, than the OR of QD. These results were statistically significant $(P<0.05)$.

\section{Associations between PI and CFS and QBYY-Q scores}

A multinomial logistic regression was used to examine associations between PI and QBYY-Q scores. The resulting values were ORs (95\% confidence intervals) with mean increments and are shown in Table 7. Using QD as a reference category for all PI scores, the $\mathrm{BD}, \mathrm{YnD}$, and YgD scale scores were positively associated with each corresponding deficiency pattern (adjusted ORs: BD-Q score $=5.877$, YnD-Q score $=12.570$, YgD-Q score $=13.558$ ).

Table 6 Crude and adjusted odds ratios of CF by PI

\begin{tabular}{lll}
\hline PI & Crude & Model 1 \\
\hline QD & Reference & Reference \\
BD & $2.49(0.88,7.85)$ & $3.41(1.10,11.87)^{*}$ \\
YnD & $2.41(0.85,7.61)$ & $3.41(1.13,11.48)^{*}$ \\
YgD & $3.74(1.19,14.40)^{*}$ & $4.68(1.35,19.65)^{*}$ \\
\hline
\end{tabular}

Odds ratio OR (95\% confidence interval), qi deficiency was used as a reference category, Model 1 adjusted for sex and age, QD qi deficiency, BD blood deficiency, YnD yin deficiency, YgD yang deficiency, Pl pattern identification

${ }^{*}<0.05,{ }^{* *}<0.01,{ }^{* * *}<0.001$

\section{Discussion}

In this study, we aimed to clinically develop a PI questionnaire and to analyze the effectiveness of the four QBYY-Q scales as diagnostic tools for CF subtypes. We examined the correlations between CF and the QBYY$\mathrm{Q}$ scales using KMD inter-rater agreement, the internal consistency and construct validity of the QBYY-Q, demographic characteristics, the convergent validity of the QBYY-Q, the least square means of the QBYY-Q scores with CF status, the associations between PIs and CF, and associations between PI and CF and QBYY-Q scores.

We compared the sociodemographic and clinical characteristics of CF patients with those of healthy subjects; however, it was difficult to delineate the clinical characteristics of CF in our study because of the small number of participants, i.e., 121 patients and 30 healthy subjects. In contrast to previous studies, the current study found no sex differences among groups, but it showed significant differences among groups for marital status and sex $[33,34]$.

Validation of the QBYY-Q and the Delphi methods were conducted using two different groups of clinicians, which strengthened the discriminant ability of the QBYYQ $[23,28]$. The revised QBYY-Q exhibited satisfactory internal consistency, with a Cronbach's $\alpha$ of 0.900 for the overall signs and symptoms, and the internal consistency of each pattern was satisfactory $(0.717-0.826)$. The strong internal consistency reliability for each pattern suggested that the pattern constructs were homogenous or that the signs and symptoms were appropriate measures of these deficiency syndrome constructs.

Table 7 Associations between PI and QBYY-Q scores

\begin{tabular}{lllccc}
\hline & & QD & BD & YnD & YgD \\
\hline Crude & QD-Q score & Reference & $0.05(0.01,0.26)^{* * *}$ & $0.17(0.02,0.61)^{* *}$ & $0.07(0.01,0.44)^{*}$ \\
& BD-Q score & Reference & $10.97(2.10,57.19)^{* *}$ & $1.25(0.25,6.32)$ & $4.99(0.90,27.60)$ \\
& YnD-Q score & Reference & $1.32(0.21,8.18)$ & $10.69(1.72,66.42)^{*}$ & $0.66(0.10,4.51)$ \\
Model 1 & YgD-Q score & Reference & $0.64(0.17,2.32)$ & $0.28(0.07,1.13)$ & $14.64(3.80,56.38)^{* * *}$ \\
& QD-Q score & Reference & $0.04(0.01,0.24)^{* * *}$ & $0.12(0.02,0.66)^{*}$ & $0.05(0.01,0.40)^{* *}$ \\
& BD-Q score & Reference & $5.88(1.04,33.12)$ & $1.07(0.20,5.79)$ & $2.39(0.38,14.18)$ \\
& YnD-Q score & Reference & $2.12(0.32,14.19)$ & $12.57(1.90,83.07)^{* *}$ & $1.22(0.16,9.20)$ \\
& YgD-Q score & Reference & $0.53(0.14,2.01)$ & $0.27(0.07,1.10)$ & $13.56(3.40,53.99)^{* * *}$ \\
\hline
\end{tabular}

Odds ratio OR (95\% confidence interval) obtained from multinomial logistic regression, qi deficiency was used as a reference category, Model 1 adjusted for sex and age, $Q D$ qi deficiency, $Q D-Q$ qi deficiency questionnaire, BD blood deficiency, BD-Q blood deficiency questionnaire, YnD yin deficiency, YnD-Q yin deficiency questionnaire, $Y g D$ yang deficiency, YgD-Q yang deficiency questionnaire, $F L$ factor loading

${ }^{*}<0.05$, ${ }^{* *}<0.01$, ${ }^{* * *}<0.001$ 
The two QD factors were identified as weakness and shortage of $q i$ or faint breathing. The two BD factors were identified as pale complexion and palpitations, i.e., a subjective sensation of rapid and forceful beating of the heart. Yin and yang deficiencies were divided into two factors each, as follows: (1) dry skin and mouth and heat vexation fever accompanied by uneasiness or restlessness for YnD and (2) and cold in the extremities up to the knees and elbows or beyond and diarrhea for YgD [35]. In summary, the $\mathrm{QD}, \mathrm{BD}, \mathrm{YnD}$, and $\mathrm{YgD}$ scores might be beneficial for clarifying the characteristics of deficiency syndromes in clinical cases.

Convergent validity was assessed by investigating the relationships between the QBYY-Q scales and the CFS-K and FSS fatigue questionnaires. The convergent validity test showed that the QBYY-Q scales were significantly correlated with CFS-K and FSS scores $(0.29<r<0.72)$.

Additionally, our study explored biochemical differences in the blood between the CF and control groups. The blood biochemistry and complete blood cell counts fell within the normal laboratory ranges [14, 35-37]. However, a CF diagnosis with YgD was significantly correlated with hemoglobin $(r=-0.28)$ and red blood cell counts $(r=-0.29)$, and a CF diagnosis with YnD was significantly correlated with chloride levels $(r=0.28)$. Statistically negative correlation coefficients of hematocrit $(r=-0.37)$, hemoglobin $(r=-0.35)$, red blood cells $(r=-0.32)$, aspartate transaminase $(r=-0.29)$, and alanine transaminase $(r=-0.28)$ were observed in CF patients with BD. These data suggest that CF patients showed differences in physiological homeostasis.

YgD of the spleen and kidney is characterized by cold limbs, listlessness, cold and pain in the waist and knee joints, a pale tongue with a white coating, and a deep and thready pulse [9]. The cross-tabulation results of CF and the PIs showed that the YgD OR (4.68, CI 1.34719.65) was significantly higher than the ORs of the other patterns (Table 6). The use of a questionnaire for deficiency syndromes might help to identify appropriate $q i$, blood, yin, and yang nourishing treatments for $\mathrm{CF}$ patients.

The YgD OR was significantly higher than the ORs of the other patterns (Table 7). However, the reliability of the YgD-Q $(\alpha=0.717)$ was lower than that of the other patterns, indicating that some of the YgD-Q items might not have accurately reflected the $\mathrm{YgD}$ pattern. In other words, the QBYY-Q did not distinguish YgD from QD because YgD simultaneously included QD. The ORs suggested that the QBYY-Q scores might be beneficial for clarifying the characteristics of deficiency syndromes in clinical cases.

The ORs derived from the multinomial logistic regression analysis demonstrated that each QD, BD, YnD, and YgD-Q score was positively associated with each corresponding deficiency pattern (adjusted ORs: qi score $=0.041$, blood score $=5.877$, yin score $=12.57$, yang score $=13.56$, reference category: QD). We constructed an algorithm for estimating the PI of a CF patient, including data on age, sex, and QBYY-Q scores, and tested its predictability (Fig. 3). We developed and validated this algorithm based on the diagnostic results and QBYY-Q scores to predict the PIs of patients with CF. To evaluate the distinction possibility of the four deficiency patterns, we developed and validated an algorithm based on diagnostic results and QBYY-Q scores. The algorithm was developed and validated based on bootstrap resampling $(B=500)$ using ordinary multinomial logistic regression (precision $=0.608$ ) and subscales of each deficiency pattern questionnaire, based on EFA (precision $=0.530$ ) [38]. The selected final model depended on the simulation results. Bootstrap validation with 500 resamples of the selected model produced moderate predictive performance, with Cox \& Snell $R^{2}=0.346$, agreement $=0.523, \mathrm{\kappa}=0.369$, area under the curve $=0.750$, precision $=0.53$, and recall $=0.535$. The prevalence and severity of ICF and CFS varied according to ethnicity and psychosocial factors [39]. By validating the results, we confirmed the possibility of diagnosis using the QBYY-Q. Objective measurement of the subjective severity of fatigue is difficult, but it is essential in implementing therapy for unexplained CF [40, 41].

The study was limited by the small number of patients and the single-center design. Although CFS and ICF can be diagnosed using international guidelines, these standards are different from PI symptoms and signs, which are very difficult to standardize. However, we generated reference data that are applicable to future studies. Thus, the results of the present study could be used to improve the care of patients with CF and CF-related disorders and to facilitate research on anti-CF therapies. Large clinical trials in multiple centers on PI and the evaluation of the therapeutic effects on $\mathrm{CF}$ in randomized clinical trials are needed. Additional studies are required to assess the correlations between CF type and other demographic and clinical characteristics.

\section{Conclusion}

The QBYY-Q was a suitable instrument for estimating the influences of qi, blood, yin, and yang deficiencies in CF. 


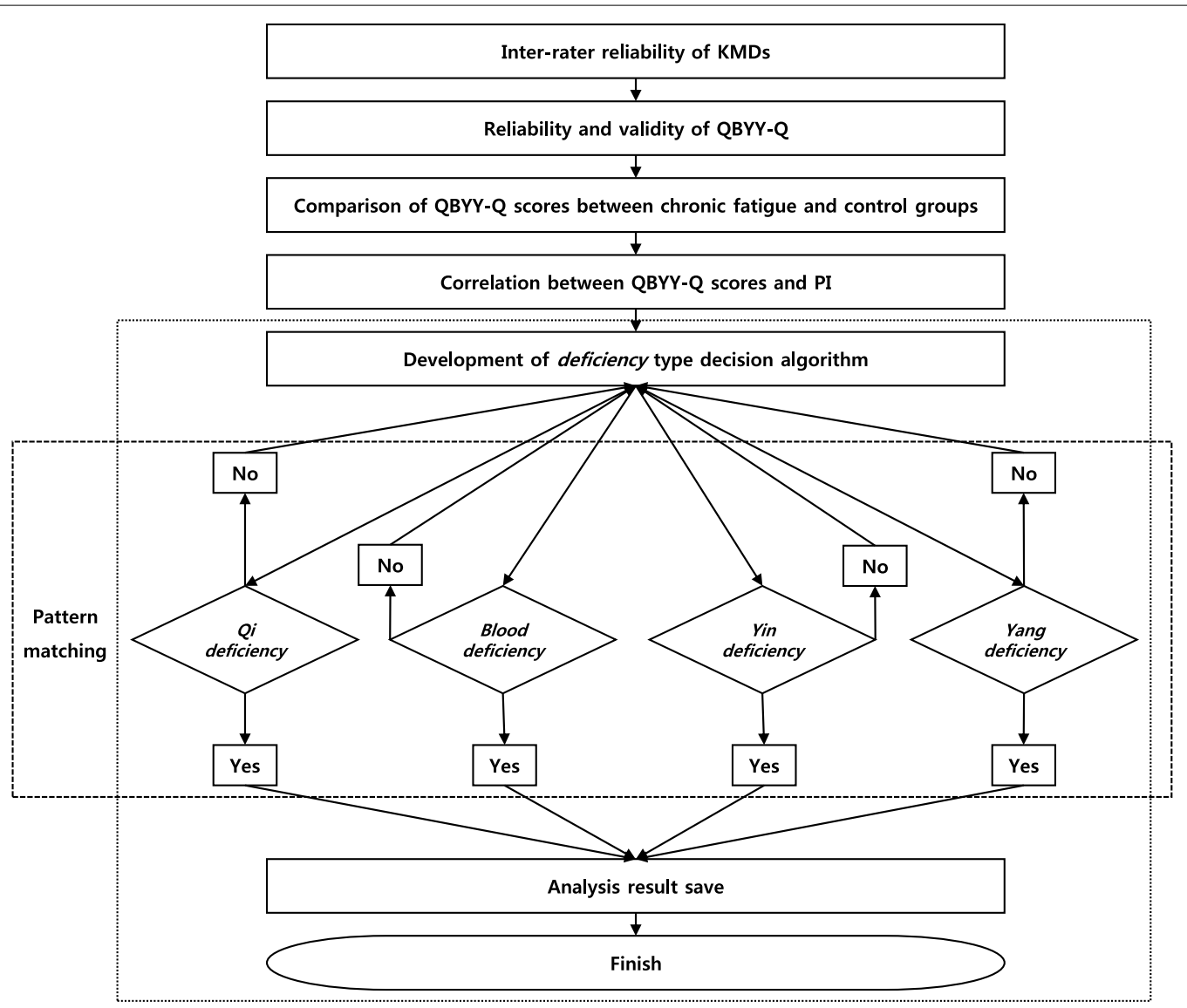

Fig. 3 Development of an algorithm for deficiency-type identification

\section{Additional files}

Additional file 1. Approval number of Clinical Research Information Service (CRIS).

Additional file 2. Informed consent form.

Additional file 3. Complaints and symptoms of Qi blood Yin Yang deficiency questionnaire

\section{Acknowledgements}

We thank all of the subjects who participated in this study and our colleagues. This study was supported by the Korea Institute of Oriental Medicine (K15011), Republic of Korea.

\section{Competing interests}

The authors declare that they have no competing interests.

Received: 9 March 2015 Accepted: 18 April 2016

Published online: 02 May 2016

\section{Abbreviations}

KM: Korean medicine; CFS: chronic fatigue syndrome; ICF: idiopathic chronic fatigue; CF: chronic fatigue; FSS: fatigue severity scale; CFS-K: Korean-translated Chalder fatigue scale; PSS: perceived stress scale; QBYY-Q: qi blood yin yang deficiency questionnaire; PI: pattern identification; KMD: Korean medical doctor; QD: qi deficiency; QD-Q: qi deficiency questionnaire; BD: blood deficiency; BD-Q: blood deficiency questionnaire; YnD: yin deficiency; YnD-Q: yin deficiency questionnaire; YgD: yang deficiency; YgD-Q: yang deficiency questionnaire; SBP: systolic blood pressure; DBP: diastolic blood pressure; KMO: Keiser Meyer Olkin; FL: factor loading; OR: odds ratio.

\section{Authors' contributions}

$\mathrm{JH}, \mathrm{BC}$ and $\mathrm{KH}$ conceived and designed the clinical trials. $\mathrm{JH}$ and $\mathrm{BC}$ analyzed the data. JH wrote the manuscript. $\mathrm{BC}$ and $\mathrm{KH}$ reviewed the manuscript. All authors read and approved the final manuscript.

\section{References}

1. Wong WS, Fielding R. Prevalence of chronic fatigue among Chinese adults in Hong Kong: a population-based study. J Affect Disord. 2010;127:248-56.

2. Jorgensen R. Chronic fatigue: an evolutionary concept analysis. J Adv Nurs. 2008;63:199-207.

3. Reeves WC, Jones JF, Maloney E, Heim C, Hoaglin DC, Boneva RS, Morrissey $M$, Devlin R. Prevalence of chronic fatigue syndrome in metropolitan, urban, and rural Georgia. Popul Health Metr. 2007:5:5.

4. Son CG. Review of the prevalence of chronic fatigue worldwide. J Korean Orien Med. 2012;33:25-33.

5. Wang YY, Li XX, Liu JP, Luo H, Ma LX, AlraekT. Traditional Chinese medicine for chronic fatigue syndrome: a systematic review of randomized clinical trials. Complem Ther Med. 2014;22:826-33. 
6. Logan AC, Wong C. Chronic fatigue syndrome: oxidative stress and dietary modifications. Altern Med Rev. 2001;6:450-9.

7. Papadopoulos AS, Cleare AJ. Hypothalamic-pituitary-adrenal axis dysfunction in chronic fatigue syndrome. Nat Rev Endocrinol. 2011;8:22-32.

8. Rosenblum H, Shoenfeld Y, Amital H. The common immunogenic etiology of chronic fatigue syndrome: from infections to vaccines via adjuvants to the ASIA syndrome. Infect Dis Clin North Am. 2011;25:851-63.

9. Chen R, Moriya J, Yamakawa J, Takahashi T, Kanda T. Traditional Chinese medicine for chronic fatigue syndrome. Evid Based Complem Alternat Med. 2010;7:3-10.

10. Afari N, Buchwald D. Chronic fatigue syndrome: a review. Am J Psychiatr. 2003;160:221-36

11. Lijue Z. Acupuncture and Chinese patent drugs for treatment of chronic fatigue syndrome. J Tradit Chin Med. 2005;25:99-101.

12. Kim JE, Hong KE, Kim HJ, Choi JB, Baek YH, Seo BK, Lee S, Kang KW, Lee MH, Kim JH, Lee S, Jung SY, Jung HJ, Shin MS, Choi SM. An open-label study of effects of acupuncture on chronic fatigue syndrome and idiopathic chronic fatigue: study protocol for a randomized controlled trial. Trials. 2013;14:147.

13. Kim JE, Seo BK, Choi JB, Kim HJ, Kim TH, Lee MH, Kang KW, Kim JH, Shin KM, Lee S, Jung SY, Kim AR, Shin MS, Jung HJ, Park HJ, Kim SP, Baek YH, Hong KE, Choi SM. Acupuncture for chronic fatique syndrome and idiopathic chronic fatigue: a multicenter, nonblinded, randomized controlled trial. Trials. 2015;16:314.

14. Zaslawki C. Clinical reasoning in traditional Chinese medicine: implications for clinical research. Clin Acupunct Orien Med. 2003;4:94-101.

15. Jiang M, Lu C, Zhang C, Yang J, Tan Y, Lu A, Chan K. Syndrome differentiation in modern research of traditional Chinese medicine. J Ethnopharmacol. 2012:140:634-42.

16. Yiu YM, Qiu MY. A preliminary epidemiological study and discussion on traditional Chinese medicine pathogenesis of chronic fatigue syndrome in Hong Kong. Zhong Xi Yi Jie He Xue Bao. 2005;3:359-62.

17. Peng M, Ma HB, Si GM. A literature review on Chinese medicine syndrome and syndrome elements of chronic fatigue syndrome. Zhongguo Zhong Xi Yi Jie He Za Zhi. 2014;34:691-3.

18. Lee NH, Son CG. Fatigue severity of patients with idiopathic chronic fatigue compared to healthy subjects. J Tradit Chin Med. 2012;32:355-7.

19. Pacific WHO Regional Office for the Western. WHO international standard terminologies on traditional medicine in the western pacific region. Virginia: World Health Organization; 2007. p. 47-55.

20. Romero-Corral A, Somers VK, Sierra-Johnson J, Thomas RJ, Collazo-Clavell ML, Korinek J, Allison TG, Batsis JA, Sert-Kuniyoshi FH, Lopez-Jimenez F. Accuracy of body mass index in diagnosing obesity in the adult general population. Int J Obes (Lond). 2008:32:959-66.

21. Organization World Health. BMI classification: The WHO Global Database on BMl; 2014. [updated 12 May 2015]. http://apps.who.int/bmi/index. jsp?introPage=intro_3.html.

22. Fukuda K, Straus SE, Hickie I, Sharpe MC, Dobbins JG, Komaroff A. The chronic fatigue syndrome: a comprehensive approach to its definition and study. International chronic fatigue syndrome study group. Ann Intern Med. 1994;121:953-9.

23. Kim JH, Ku BC, Kim YS, Kim KH. Study on reliability and validity of the 'qi blood Yin Yang deficiency questionnaire. Korean J Orien Physiol Pathol. 2014;28:346-54.

24. Wang H, Liu X, Lv B, Yang F, Hong Y. Reliable multi-label learning via conformal predictor and random forest for syndrome differentiation of chronic fatigue in traditional Chinese medicine. PLoS ONE. 2014;9:e99565.
25. Park YJ, Cho SW, Lee BH, Park YB. Development and validation of the Yin deficiency scale. J Altern Complem Med. 2013;19:50-6.

26. Berle CA, Cobbin D, Smith N, Zaslawski C. A novel approach to evaluate Traditional Chinese Medicine treatment outcomes using pattern identification. J Altern Complem Med. 2010;16:357-67.

27. Feng Z, Pang Y, Ye FW. TCM non-medicinal therapy for regulation and treatment of chronic fatigue syndrome. J Acupun Tuina Sci. 2008:6:376-80

28. Woo HJ, Kim SH, Lee SB, Choi MY, Kim YC, Lee JH. Development of Questionnaires for Differentiation of qì-xū, xuè-xū, yang-xū, ȳ̄n-xū analysis. Korean J Orient Int Med. 2008:29:856-70.

29. Krupp LB, LaRocca NG, Muir-Nash J, Steinberg AD. The fatigue severity scale. Application to patients with multiple sclerosis and systemic lupus erythematosus. Arch Neurol. 1989:46:1121-3.

30. Chalder T, Berelowitz G, Pawlikowska T, Watts L, Wessely S, Wright D, Wallace EP. Development of a fatigue scale. J Psychosom Res. 1993;37:147-53.

31. Ihaka R, Gentleman R. R: a language for data analysis and graphics. J Comput Graphic Stat. 1996;5:299-314.

32. Ferrari E, Veschi M, Guerzoni V, Bergamini L, Neviani F, Manni B, Pelosi A Neri M. A scale evaluating the emotivity and well-being of elderly (Italian acronym: SVEBA): analysis of psychometric characteristics. Arch Gerontol Geriatr. 2009:49:77-81.

33. Kim $\mathrm{CH}$, Shin $\mathrm{HC}$, Won CW. Prevalence of chronic fatigue and chronic fatigue syndrome in Korea: community-based primary care study. J Korean Med Sci. 2005;20:529-34.

34. Buchwald D, Pearlman T, Kith P, Schmaling K. Gender differences in patients with chronic fatigue syndrome. J Gen Intern Med. 1994:9:397-401.

35. Lee BK, Kim TH, Park YB. Korean medicine diagnosis theory. 7th ed. Seoul: Seongbosa company press; 2009. p. 312-7.

36. Swanink CM, Vercoulen JH, Bleijenberg G, Fennis JF, Galama JM, van der Meer JW. Chronic fatigue syndrome: a clinical and laboratory study with a well matched control group. J Intern Med. 1995;237:499-506.

37. Niblett SH, King KE, Dunstan RH, Clifton-Bligh P, Hoskin LA, Roberts TK, Fulcher GR, McGregor NR, Dunsmore JC, Butt HL, Klineberg I, Rothkirch TB. Hematologic and urinary excretion anomalies in patients with chronic fatigue syndrome. Exp Biol Med (Maywood). 2007;232:1041-9.

38. Efron B, Tibshirani RJ. An introduction to the bootstrap. Boca Raton: CRC Press; 1994.

39. Mawle AC, Nisenbaum R, Dobbins JG, Gary HE Jr, Stewart JA, Reyes M, Steele L, Schmid DS, Reeves WC. Immune responses associated with chronic fatigue syndrome: a case-control study. J Infect Dis. 1997:175:136-41.

40. Dinos S, Khoshaba B, Ashby D, White PD, Nazroo J, Wessely S, Bhui KS. A systematic review of chronic fatique, its syndromes and ethnicity: prevalence, severity, co-morbidity and coping. Int J Epidemiol. 2009;38:1554-70

41. Whitehead L. The measurement of fatigue in chronic illness: a systematic review of unidimensional and multidimensional fatigue measures. J Pain Symptom Manag. 2009;37:107-28.

\section{Submit your next manuscript to BioMed Central and we will help you at every step:}

- We accept pre-submission inquiries

- Our selector tool helps you to find the most relevant journal

- We provide round the clock customer support

- Convenient online submission

- Thorough peer review

- Inclusion in PubMed and all major indexing services

- Maximum visibility for your research

Submit your manuscript at www.biomedcentral.com/submit
BioMed Central 